\title{
Challenging False Discovery Rate: A Partition Test Based on $p$ Values in Human Case-Control Association Studies
}

\author{
Jurg Ott ${ }^{\mathrm{a}}$ Zhe Liu ${ }^{\mathrm{b}}$ Yuanyuan Shen ${ }^{\mathrm{c}}$ \\ ${ }^{a}$ Key Laboratory of Mental Health, Institute of Psychology, CAS, Beijing, China; Department of Statistics, University of \\ Chicago, Chicago, III., and 'Department of Biostatistics, Harvard School of Public Health, Boston, Mass., USA
}

\section{Key Words}

Multilocus analysis - Case-control association test •

False discovery rate $\cdot$ Multiple testing

\begin{abstract}
Background/Aims: We consider the situation that multiple genetic variants are underlying a heritable trait and assume that each contributes to the trait only to a small degree. The aim is to develop a statistical test for disease association of these multiple variants. Methods: We expect that $p$ values resulting from a genome-wide case-control association analysis will fall into two classes: those reflecting true association and those occurring randomly in the interval from 0 to 1. We develop a partition test to find the set of smallest $p$ values deviating most from the number of $p$ values expected under randomness. Results: Power calculations demonstrate the superiority of our partition test over conventional SNP-by-SNP analyses. Applications of the partition test to six published datasets show that our test is particularly suitable when multiple SNPs appear to contribute to a trait, and furnished more significant results than a well-known procedure to estimate the false discovery rate. Conclusions: Our partition test also furnishes an estimate of the number of functional SNPs underlying disease and can be highly significant, while single-locus tests may be far from significant.
\end{abstract}

(C) 2012 S. Karger AG, Basel

$0001-5652 / 12 / 0741-0045 \$ 38.00 / 0$

Fax +41613061234

E-Mail karger@karger.ch

www.karger.com
Accessible online at: www.karger.com/hhe

\section{Introduction}

Common heritable traits are often thought to be due to a number of functional variants that may interact with each other and the environment to lead to disease. Consequently, in human case-control association studies, single-nucleotide polymorphisms (SNPs) in the vicinity of functional variants may only show moderate degrees of association. To determine, which among the generally large number, $m$, of SNPs tested for association is a true positive, represents a statistical challenge. One answer to this multiple testing problem [1] has been to limit the experiment-wise (genome-wide) significance level, $\alpha=$ $\mathrm{P}$ (one or more test results exceed a threshold, $T$, given no association), where $T$ is the statistical threshold for nominal significance at each SNP, with $p=\mathrm{P}$ (test result $\geq T$ for a given SNP when there is no association). The Bonferroni correction for multiple testing, $\alpha=p \times m$ with $\alpha \leq 0.05$, is based on such considerations but is known to be generally rather conservative. A less conservative solution is to determine an equivalent number, $m^{\prime}<m$, of independent SNPs and apply the Bonferroni correction with $m$ ' instead of $m[2,3]$.

As an alternative to the classical concept of statistical significance, it has been proposed that significance be based on the conditional probability, $\mathrm{P}$ (test result is false given it is significant). This probability is the false discov- 
Table 1. Results of power simulations based on 1,000 simulated datasets

\begin{tabular}{llll}
\hline SNPs, n & $\begin{array}{l}\text { Definition } \\
\text { of success }\end{array}$ & $\begin{array}{l}\text { Partition } \\
\text { test }\end{array}$ & $\begin{array}{l}\text { SNP-specific } \\
\text { allele test }\end{array}$ \\
\hline 1 & Detect 1 SNP & 0.252 & 0.246 \\
5 & Detect $\geq 1$ SNP & 0.828 & 0.759 \\
5 & Detect $\geq 3$ SNPs & 0.287 & 0.125 \\
10 & Detect $\geq 1$ SNP & 0.981 & 0.942 \\
10 & Detect $\geq 5$ SNPs & 0.223 & 0.086 \\
20 & Detect $\geq 1$ SNP & 1.000 & 0.998 \\
20 & Detect $\geq 5$ SNPs & 0.771 & 0.606 \\
\hline
\end{tabular}

ery rate (FDR) [4], and limiting it to no more than 0.05 often achieves more significant results than limiting $\alpha$. Assessing FDRs requires determining whether a test result is true or false, and this can be estimated in a number of different ways [5-8], each resulting in somewhat different FDR values. One of the main aspects of the FDR concept is that it works on nominal $p$ values. To avoid the ambiguities inherent in FDR, we take advantage of the concept of working with p values but develop a significance test in the classical statistical sense. Specifically, for an ordered set of $p$ values, we develop a partition test whose significance level is assessed in permutation samples.

\section{Partition Test}

Recursive partitioning is a popular clustering method to recursively divide a set of items into successively more homogeneous groups $[9,10]$. Such clustering is generally carried out in an ad hoc manner. For example, haplotypes at the $\mu$ opioid receptor gene were determined in 250 substance-dependent individuals and controls and recursively partitioned based on sequence similarity [11]. At each step, a statistical test was carried out to assess, in the different clusters, differences in haplotype frequencies among cases and controls, and that set of clusters furnishing the smallest $p$ value was of most interest. Rigorous statistical analysis must take this search for the smallest $\mathrm{p}$ value into account, for example, by treating it as a test statistic [12]. Here, we make use of this concept to develop a partition test for genome-wide case-control association (GWA) studies.

Consider a number $m$ of SNPs and a set of cases and controls genotyped at each of the $m$ SNPs. Initial SNP-specific statistical tests furnish $m \mathrm{p}$ values, of which a presumably small number represent true positive findings. We wish to find the set of (small) $\mathrm{p}$ values representing true association. Thus, we define a threshold, $t_{\mathrm{p}}$, and count the number of $\mathrm{p}$ values falling below $t_{\mathrm{p}}$ and those at or above $t_{\mathrm{p}}$. In the absence of any association (null hypothesis, $\mathrm{H}_{0}$ ), the $\mathrm{p}$ values follow a uniform $\mathrm{U}(0,1)$ distribution, so the expected proportion of $\mathrm{p}$ values below $t_{\mathrm{p}}$ is equal to $t_{\mathrm{p}}$. As a measure of discrepancy between observed and expected numbers of $p$ values in the two classes defined by $t_{\mathrm{p}}$, we use the customary $\chi^{2}$ statistic, $X^{2}$, but set it equal to zero when the observed number of small $\mathrm{p}$ values is less than the corresponding expected number. As these $\mathrm{p}$ values are likely to be non-independent, $X^{2}$ does not follow the $\chi^{2}$ distribution with 1 d.f. even under $\mathrm{H}_{0}$, but at this point $X^{2}$ is merely a measure of discrepancy.

As we are unsure of the appropriate setting of $t_{\mathrm{p}}$ without looking at the data, we position $t_{\mathrm{p}}$ at a reasonable upper level such as $t_{\mathrm{u}}=0.10$, and then let $t_{\mathrm{p}}$ take a number $k$ of equal steps from $t_{\mathrm{u}}$ down to zero. For example, with $k=1,000$ and $t_{\mathrm{u}}=0.10$, step size is $0.10 / 1,000=0.0001$, which is also the smallest value $t_{\mathrm{p}}$ will take. At each step, we determine $X^{2}$ and record the largest result, $X^{2}$ max which will serve as our (genome-wide) test statistic. To test $\mathrm{H}_{0}$ against the alternative of an excess of small $p$ values, rather than trying to find the theoretical null distribution of $X^{2}{ }_{\max }$ [13], we estimate it by randomization: we randomly permute the labels case and control but leave genotypes untouched. We do this $r$ times. In each of the resulting $r$ permutation datasets, we carry out the same procedure as for the observed data, that is, find a value of $X^{2}$ max wherever it occurs between $\mathrm{p}=0$ and $\mathrm{p}=t_{\mathrm{u}}$. The proportion of permutation samples with $X^{2}{ }_{\max }$ at least as large as the one in the observed data estimates the empirical significance level associated with the observed $X^{2}$ max. This significance level is unbiased and can be as accurate as desired depending on the number, $r$, of permutation datasets [14].

In our partition test, it is not only of interest whether it furnishes significant results. The threshold, $t_{\mathrm{p}}$, estimated by $X_{\text {max }}^{2}$, also represents a key result as it allows a rough estimate of the number of SNPs with true disease association, that is, the number, $m_{\mathrm{t}}$, of SNPs below the threshold $t_{\mathrm{p}}$ associated with $X^{2}{ }_{\text {max }}$ (more on this in the Discussion).

\section{Power Simulations}

To compare performance of our partition test versus the customary single-locus $\chi^{2}$ test based on allele frequencies, we carried out the following computer simulation. We generated $r=1,000$ replicates of case-control data, each with 200 cases and 200 controls and 1,000 SNPs (independent of each other). Four disease models were considered: (1) 1 disease-associated SNP, 999 noncausal SNPs; (2) 5 disease-associated SNPs, 995 non-causal SNPs; (3) 10 disease-associated SNPs, 990 non-causal SNPs, and (4) 20 disease-associated SNPs, 980 non-causal SNPs. In each scenario, recessive inheritance was assumed for the disease-associated SNPs, which were taken to be the functional disease variants with an odds ratio of 3.0. The minor allele frequency at each SNP was set equal to 0.30 and disease prevalence was taken to be $5 \%$.

In each replicate, our partition test and the single-locus $\chi^{2}$ allele test were applied. For the partition test, we assumed $t_{\mathrm{u}}=0.05$ with $k=1,000$ steps. To ensure a size of 0.05 for each of the two tests, we generated 1,000 replicates under $\mathrm{H}_{0}$ (no causal SNPs) and chose a threshold for each test statistic (the largest $\chi^{2}$ for the allele test) such that the proportion of 'significant' outcomes was equal to 0.05 . As table 1 shows, our partition test clearly outperforms the SNP specific test, particularly for detecting more than one causal SNP. 
Table 2. Published case-control datasets analyzed

\begin{tabular}{|c|c|c|c|c|c|c|c|}
\hline \multirow[t]{2}{*}{ Dataset } & \multirow{2}{*}{$\begin{array}{l}\text { SNPs } \\
\mathrm{n}\end{array}$} & \multirow{2}{*}{$\begin{array}{l}\text { cases } \\
\mathrm{n}\end{array}$} & \multirow{2}{*}{$\begin{array}{l}\text { controls } \\
\mathrm{n}\end{array}$} & \multicolumn{2}{|c|}{ Partition test } & \multicolumn{2}{|c|}{ Single-locus test } \\
\hline & & & & $\mathrm{p}$ & $m_{\mathrm{t}}$ & $\min . p$ & $\min . F D R$ \\
\hline Age-related macular degeneration & 81,934 & 96 & 127 & 0.2307 & 180 & $2.12 \times 10^{-7}$ & $2.11 \times 10^{-7}$ \\
\hline Schizophrenia & 711,484 & 921 & 954 & 0.0001 & 12,582 & 0.8535 & 0.6160 \\
\hline Parkinson's disease & 379,502 & 270 & 271 & 0.0168 & 132 & 0.2045 & 0.2288 \\
\hline Rheumatoid arthritis & 338,671 & 868 & 1,194 & 0.0001 & 1,299 & 0.0008 & 0.0008 \\
\hline Response to chemotherapy & 302,865 & 75 & 170 & 0.0503 & 38 & 0.4920 & 0.3506 \\
\hline Heroin addiction & 9,335 & 100 & 100 & 0.0529 & 11 & 0.1951 & 0.2171 \\
\hline
\end{tabular}

$\mathrm{p}=$ Empirical significance level, corrected for multiple testing; $m_{\mathrm{t}}=$ number of SNPs with SNP-specific $\mathrm{p}$ values smaller than threshold leading to $X^{2}{ }_{\text {max }}$; min.p = smallest Bonferroni-corrected $\mathrm{p}$ value; $\min . \mathrm{FDR}=$ smallest false discovery rate.

\section{Application of the Partition Test to Observed Datasets}

Rather than carrying out more extensive power simulations under possibly unrealistic inheritance models, we applied our approach to six datasets from published GWA studies. Each analysis was based on $\chi^{2}$ test statistics in 2 $\times 2$ tables, with rows corresponding to cases and controls, and columns referring to two SNP alleles. Thus, as in our power calculations, the $\chi^{2}$ test was a standard 'allele test', in which alleles rather than individuals are counted. Parameter settings were as follows: largest threshold for $\mathrm{p}, t_{\mathrm{u}}=0.10$; number of steps, $k=1,000$; and a number of permutation replicates of 10,000 . We included the observed result as one of the null datasets [14] so that, for the permutation-based $\mathrm{p}$ values in the partition test, the smallest possible significance level is $\mathrm{p}=$ $1 / 10,001=0.0001$.

All analyses were carried out under genetic control. That is, the genomic control parameter, $\lambda$, was estimated from SNP-specific $\chi^{2}$ tests in $2 \times 2$ tables as the observed median of $\chi^{2}$ in all $\chi^{2}$ tests genome-wide divided by the corresponding theoretical median of 0.455 of the $\chi^{2}$ distribution with 1 d.f. In the presence of large numbers of functional SNPs, it has been shown [15] that an increase of $\lambda$ over its null value of 1 must be expected even with perfect homogeneity of the data. Thus, application of the genomic control procedure will lead to conservative results, at least in conventional SNP-by-SNP analyses, for which it was designed.

In our partition test, it is not only of interest whether it furnishes significant results. The threshold, $t_{\mathrm{p}}$, estimated by $X_{\text {max }}^{2}$, also represents a key result in that it allows a rough estimate of the number of SNPs with true disease association, that is, the number, $m_{\mathrm{t}}$, of SNPs below the threshold $t_{\mathrm{p}}$ associated with $X^{2}{ }_{\text {max }}$ (more on this in the Discussion). The datasets listed below were analyzed and furnished the following results (table 2).

Age-Related Macular Degeneration in Hong Kong [16]

The partition test was far from significant $(\mathrm{p}=0.2307)$ so that the number, $m_{\mathrm{t}}$, of SNPs below the $\mathrm{p}$ value furnishing $X_{\text {max }}^{2}$ is not very relevant. This trait seems to involve only a small number of functional variants, which may be the reason that single-SNP analysis is much more successful: the largest $\chi^{2}$ for a single SNP is highly significant $(\mathrm{p}<0.0001)$.

\section{Schizophrenia Dataset Obtained from the Database} of Genotype and Phenotype at National Institute of Health

Here, the situation is just reversed compared with agerelated macular degeneration: no single SNP furnished a significant result, while our partition test is highly significant $(p=0.0001)$. The number of SNPs below that value of $\mathrm{p}$ furnishing $X_{\text {max }}^{2}$ is $m_{\mathrm{t}}=12,582$. Although this may be a large estimate for the number of functional SNPs involved in schizophrenia, it confirms previously published results indicating the polygenic nature of this trait [17].

\section{Parkinson's Disease [18]}

Here, the situation is analogous to that in the schizophrenia dataset: no single SNP furnishes a significant result, but our partition test is significant $(\mathrm{p}=0.0168)$ and indicates a number of $m_{\mathrm{t}}=132$ disease SNPs. 


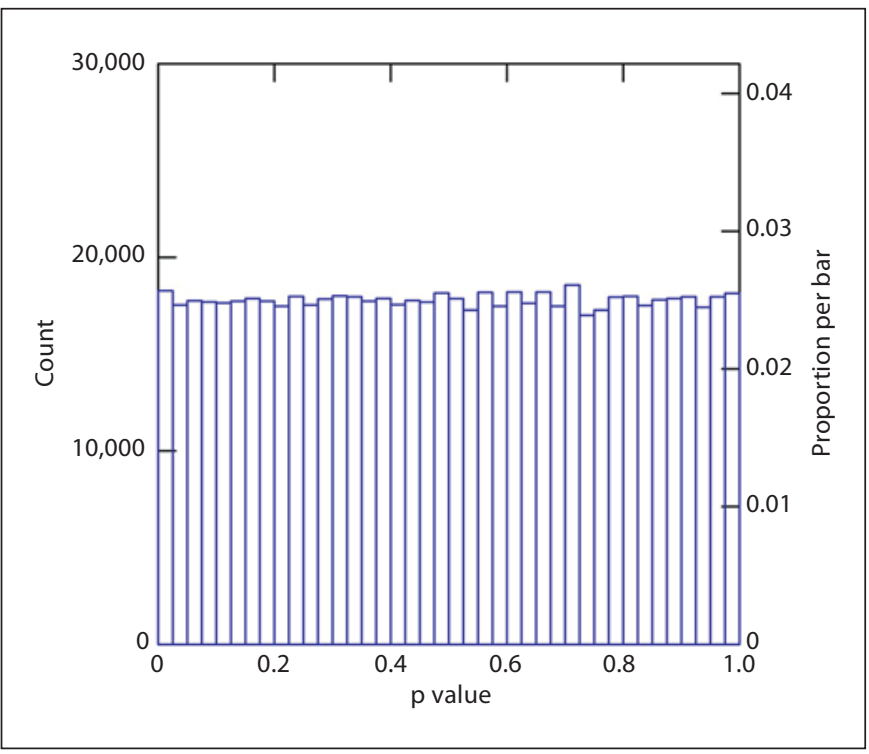

Fig. 1. Histogram of 711,484 p values for the allelic association test in the schizophrenia dataset analyzed here.

\section{Rheumatoid Arthritis [19]}

This dataset furnishes highly significant results both for single SNPs (mostly in the HLA region; details not shown here) and for our partition test, which estimates close to 1,300 polygenes.

\section{Response to Platinum-Based Chemotherapy [20]}

Here, the situation is again analogous to that in schizophrenia: results are far from significant in single-locus tests but close to significant ( $\mathrm{p}=0.0503$ ) in our partition test. A total of $m_{\mathrm{t}}=38$ polygenes are indicated.

\section{Heroin Addiction [21]}

Although not formally significant, our partition test furnishes a stronger result $(\mathrm{p}=0.0529)$ than any singlelocus test (0.1951).

\section{Discussion}

Much interest has in recent years revolved around analyzing all $\mathrm{p}$ values resulting from large-scale case-control association studies. As mentioned above, one result of this interest has been the development of the FDR concept. Our own efforts on working with multiple SNPs have 10 years ago led to the development of sum statistics $[22,23]$. Here, we propose a statistical hypothesis testing approach to analyzing all $\mathrm{p}$ values. Specifically, we test which proportion of small $p$ values yields the largest deviation (in terms of $\chi^{2}$ ) from that proportion expected based on the uniform distribution. In several of the six datasets analyzed, our partition test furnishes highly significant results while single-SNP analyses remain nonsignificant.

For each of the six published datasets analyzed here, we also estimated the smallest FDR, which was done by the q value method [7]; the Benjamini-Hochberg method [24] furnished essentially the same FDR results (not shown here). Clearly, there are strong similarities between our partition test and the FDR approach: both try to distinguish between true and false positive results, and both do this by focusing on small $p$ values as representing true association signals. Statistically, the main difference is that our partition test works under the null hypothesis of no association while FDR approaches assume, among significant results, some truly and some falsely significant results. Often, the FDR approach yields smaller values than Bonferroni-corrected $p$ values. Most remarkably, however, our partition approach performed much better than the FDR approach in five of the six datasets (table 2). While more work will be required to investigate this situation, we interpret these findings as follows: the FDR approach assumes, as $p$ values get smaller, a distinct upswing of their frequencies (see fig. 1 in [7]). In other words, the histogram of $\mathrm{p}$ values is assumed to be flat towards the right end (values approaching 1) and showing higher and higher bars towards the left (values approaching 0 ). However, with large numbers of susceptibility variants, there is no clearly perceptible increase in the frequency of small $p$ values. For example, the histogram for the $\mathrm{p}$ values in the schizophrenia dataset is essentially flat (fig. 1), and very similar histograms are obtained for most of the datasets analyzed here. Thus, while the FDR approach tends to be powerful for small numbers of susceptibility variants, we tentatively conclude that it is far less powerful than our partition test with large numbers of functional SNPs.

A suitable model underlying such considerations is that $p$ values represent two types of results: (1) those reflecting a true association with disease and (2) those due to random association. The two types of $\mathrm{p}$ values presumably form two overlapping distributions with (1) having a mean lower than (2). Such mixture distributions have previously been proposed for the analysis of microarray gene expression data $[25,26]$, and, specifically, to estimate the FDR [8]. In our partition test, we estimate that threshold $t_{\mathrm{p}}$ leading to the largest $\chi^{2}$ measuring the discrepancy 
between observed and expected numbers of small $\mathrm{p}$ values. We assume that this threshold is in some sense the 'best' dividing line between types (1) and (2) of p values. Thus, we interpret the number $m_{\mathrm{t}}$ of $\mathrm{p}$ values below this best threshold as being of type (1), that is, p values reflecting a true association. For polygenic traits, such as schizophrenia, with presumably large numbers of underlying functional variants, purely statistical analyses are unlikely to correctly identify a large portion of these variants. Bioinformatics analyses may then be helpful, for example, by assigning the number $m_{\mathrm{t}}$ of SNPs with small p values to genes and to make sense of these genes. But such approaches go beyond the scope of this investigation.

A functional variant may exhibit a small $p$ value because it has a large effect size, which is a function of the minor allele frequency and the odds ratio in a logistic model [27], or it may be small due to chance. Here, we do not work with effect sizes to estimate numbers of truly associated variants but base our analysis strictly on the distribution of $p$ values and its deviation from the uniform distribution expected under no association.

A curious aspect of our partition test is that it can be more significant with a genomic control factor, $\lambda$, exceeding 1 as opposed to $\lambda$ being equal to 1 , which is not possible in single-SNP association tests. This happened for two (Parkinson's disease and heroin addiction) of the six published datasets analyzed here (details not shown). In such cases, evidently, the increased $\lambda$ value leads to a better discrimination between $p$ values of types (1) and (2), presumably by making large $\mathrm{p}$ values adhere better to the uniform distribution.

For schizophrenia, statistical modeling predicted early on the presence of a single major gene acting on a poly- genic background $[28,29]$. Initially, gene mapping efforts focused on the major gene aspect of this prediction, but strong evidence for polygenes and non-Mendelian variation has since emerged [17, 30]. In the absence of significant single-SNP association results and biological evidence for genetic effects in complex traits, our partition test can nonetheless provide significant evidence for the polygenic nature of this trait. Such a result then at least confirms estimates of high heritability.

Finally, as pointed out by one of the reviewers, our test relies on the $\mathrm{p}$ values from statistical tests, not the test statistics themselves. Thus, it is important for users of this test to ensure that $p$ values are reasonable and valid. For example, SNPs strongly out of Hardy-Weinberg equilibrium should not be assessed with an allele-based statistical test, or should be removed prior to analysis, which presumably is the standard approach.

\section{Acknowledgments}

Support from China NSFC grant No. 30730057 is gratefully acknowledged. This study used data from the SNP Database at the NINDS Human Genetics Resource Center DNA and Cell Line Repository (http://ccr.coriell.org/ninds), as well as clinical data. The original genotyping was performed in the laboratories of Drs. Singleton and Hardy, (NIA, LNG), Bethesda, Md., USA. Funding support for development of a novel analysis methods was provided by the Rockefeller University, based on a subcontract from the Albert Einstein College of Medicine, and the genotyping of samples was provided through the Genetic Association Information Network (GAIN). The schizophrenia dataset used for the analyses described in this study was obtained from the database of Genotype and Phenotype (dbGaP) found at http://www.ncbi.nlm.nih. gov/gap. We thank Dr. P.K. Gregersen for allowing us to use his RA dataset for this study.

\section{References}

1 Zhang Q, Ott J: Multiple comparisons/testing issues; in Lin S, Zhao H (eds): Handbook on Analyzing Human Genetic Data: Computational Approaches and Software. Berlin, Springer, 2009, vol 1, pp 277-287.

2 Cheverud JM: A simple correction for multiple comparisons in interval mapping genome scans. Heredity 2001;87:52-58.

3 Nyholt DR: A simple correction for multiple testing for single-nucleotide polymorphisms in linkage disequilibrium with each other Am J Hum Genet 2004;74:765-769.

4 Benjamini Y: Discovering the false discovery rate. J R Statist Soc B 2010;72:405-416.

5 Hochberg Y, Benjamini Y: More powerful procedures for multiple significance testing. Stat Med 1990;9:811-818.
6 6 Benjamini Y, Yekutieli D: The control of the false discovery rate in multiple testing under dependency. Ann Statist 2001;29:1165-1188.

7 Storey JD, Tibshirani R: Statistical significance for genomewide studies. Proc Natl Acad Sci USA 2003;100:9440-9445.

-8 Efron B: Large-scale simultaneous hypothesis testing: the choice of a null hypothesis. Am Stat Assoc 2004;99:96-104.

9 Zhang H, Singer B: Recursive Partitioning in the Health Sciences. New York, Springer, 1999.

10 Strobl C, Malley J, Tutz G: An introduction to recursive partitioning: rationale, application, and characteristics of classification and regression trees, bagging, and random forests. Psychol Methods 2009;14:323-348.
-11 Hoehe MR, Kopke K, Wendel B, Rohde K, Flachmeier C, Kidd KK, Berrettini WH, Church GM: Sequence variability and candidate gene analysis in complex disease: association of mu opioid receptor gene variation with substance dependence. Hum Mol Genet 2000;9:2895-2908.

12 Levenstien MA, Yang Y, Ott J: Statistical significance for hierarchical clustering in genetic association and microarray expression studies. BMC Bioinformatics 2003;4:62.

13 Chotai J: Isotonic inference for populations related to the uniform distribution. Commun Statist Theory Methods 1983;12:2109_ 2118 . 
14 Manly BFJ: Randomization, Bootstrap, and Monte Carlo Methods in Biology, ed 3. Boca Raton, FL, Chapman and Hall/CRC, 2007.

15 Yang J, Weedon MN, Purcell S, Lettre G, Estrada K, Willer CJ, Smith AV, Ingelsson E, O’Connell JR, Mangino M, Mägi R, Madden PA, Heath AC, Nyholt DR, Martin NG, Montgomery GW, Frayling TM, Hirschhorn JN, McCarthy MI, Goddard ME, Visscher PM, GIANT Consortium: Genomic inflation factors under polygenic inheritance. Eur J Hum Genet 2011;19:807-812.

16 Dewan A, Liu M, Hartman S, Zhang SS, Liu DT, Zhao C, Tam PO, Chan WM, Lam DS, Snyder M, Barnstable C, Pang CP, Hoh J: HTR1 promoter polymorphism in wet agerelated macular degeneration. Science 2006; 314:989-992.

17 Purcell SM, Wray NR, Stone JL, Visscher PM, O’Donovan MC, Sullivan PF, Sklar P: Common polygenic variation contributes to risk of schizophrenia and bipolar disorder. Nature 2009;460:748-752.

-18 Fung HC, Scholz S, Matarin M, Simon-Sanchez J, Hernandez D, Britton A, Gibbs JR, Langefeld C, Stiegert ML, Schymick J, Okun MS, Mandel RJ, Fernandez HH, Foote KD, Rodriguez RL, Peckham E, De Vrieze FW, Gwinn-Hardy K, Hardy JA, Singleton A: Genome-wide genotyping in Parkinson's disease and neurologically normal controls: first stage analysis and public release of data. Lancet Neurol 2006;5:911-916.
19 Amos CI, Chen WV, Lee A, Li W, Kern M, Lundsten R, Batliwalla F, Wener M, Remmers E, Kastner DA, Criswell LA, Seldin MF, Gregersen PK: High-density SNP analysis of 642 Caucasian families with rheumatoid arthritis identifies two new linkage regions on $11 \mathrm{p} 12$ and 2q33. Genes Immun 2006; 7:277286.

20 Wu C, Xu B, Yuan P, Ott J, Guan Y, Liu Y, Liu Z, Shen Y, Yu D, Lin D: Genome-wide examination of genetic variants associated with response to platinum-based chemotherapy in patients with small-cell lung cancer. Pharmacogenet Genomics 2010;20:389395.

21 Nielsen DA, Ji F, Yuferov V, Ho A, Chen A, Levran O, Ott J, Kreek MJ: Genotype patterns that contribute to increased risk for or protection from developing heroin addiction. Mol Psychiatry 2008;13:417-428.

22 Hoh J, Wille A, Ott J: Trimming, weighting, and grouping SNPS in human case-control association studies. Genome Res 2001;11: 2115-2119.

23 Hoh J, Ott J: Mathematical multi-locus approaches to localizing complex human trait genes. Nat Rev Genet 2003;4:701-709.

24 Benjamini Y, Drai D, Elmer G, Kafkafi N, Golani I: Controlling the false discovery rate in behavior genetics research. Behav Brain Res 2001;125:279-284.
25 Allison DB, Gadbury GL, Heo M, Fernandez JR, Lee C-K, Prolla TA, Weindruch R: A mixture model approach for the analysis of microarray gene expression data. Comput Stat Data An 2002;39:1-20.

26 Lai Y: A moment-based method for estimating the proportion of true null hypotheses and its application to microarray gene expression data. Biostatistics 2007;8:744-755.

27 Park JH, Wacholder S, Gail MH, Peters U, Jacobs KB, Chanock SJ, Chatterjee N: Estimation of effect size distribution from genome-wide association studies and implications for future discoveries. Nat Genet 2010; 42:570-575.

28 Risch N, Baron M: Segregation analysis of schizophrenia and related disorders. Am J Hum Genet 1984;36:1039-1059.

29 Risch N: Linkage strategies for genetically complex traits. I. Multilocus models. Am J Hum Genet 1990;46:222-228.

30 Walsh T, McClellan JM, McCarthy SE, Addington AM, Pierce SB, Cooper GM, Nord AS, Kusenda M, Malhotra D, Bhandari A, Stray SM, Rippey CF, Roccanova P, Makarov V, Lakshmi B, Findling RL, Sikich L, Stromberg T, Merriman B, Gogtay N, Butler P, Eckstrand K, Noory L, Gochman P, Long R, Chen Z, Davis S, Baker C, Eichler EE, Meltzer PS, Nelson SF, Singleton AB, Lee MK, Rapoport JL, King MC, Sebat J: Rare structural variants disrupt multiple genes in neurodevelopmental pathways in schizophrenia. Science 2008;320:539-543. 